\title{
Immunoinformatics Approach to Design T-cell Epitope-Based Vaccine Against Hendra Virus
}

\author{
Mohit Kamthania $^{1,2} \cdot$ Sukrit Srivastava $^{2} \cdot$ Meha Desai $^{3} \cdot$ Anubhav Jain $^{1} \cdot \operatorname{Archana~Shrivastav~}^{4} \cdot$ D. K. Sharma ${ }^{1}$
}

Accepted: 29 December 2018 / Published online: 9 January 2019

(c) Springer Nature B.V. 2019

\begin{abstract}
Screening of HLA class II epitope-based peptides as potential vaccine candidates is one of the most rational approach for vaccine development against Hendra virus $(\mathrm{HeV})$ infection, for which currently there is no successful vaccine in practice. In this study, screening of epitopes from $\mathrm{HeV}$ proteins viz matrix, glycoprotein, nucleocapsid, fusion, $\mathrm{C}$ protein, $\mathrm{V}$ protein, $\mathrm{W}$ protein and polymerase, followed by highest binding affinity \& molecular dynamic simulation of selected T-cell epitopes with their corresponding HLA class II alleles has been done. The server ProPred facilitates the binding prediction of HLA class II allele specific epitopes from the antigenic protein sequences of HeV. PEPstrMOD server was used for PDB structure modeling of the screened epitopes and MODELLER was used for HLA alleles modeling. We docked the selected T-cell epitopes with their corresponding HLA allele structures using the AutoDock 4.2 tool. Further the selected docked complex structures were optimized by NAnoscale Molecular Dynamics program (NAMD) at $5 \mathrm{ps}$, with the CHARMM-22 force field parameter incorporated in Visual Molecular Dynamics (VMD 1.9.2) and complex structure stability was evaluated by calculating RMSD values. Epitopes IRIFVPATN (Nucleocapsid), MRNLLSQSL (Nucleocapsid), VRRAGKYYS (Matrix) and VRLKCLLCG (Fusion) proteins have shown considerable binding with DRB1*0806, DRB 1*1304, DRB1*0701 and DRB1*0301 HLA class II allele respectively. Toxicity, antigenicity and population coverage of epitopes IRIFVPATN, MRNLLSQSL, VRRAGKYYS and VRLKCLLCG were analyzed by Toxin Pred, Vexijen and IEDB tool, respectively. The potential T-cell epitopes can be utilized in designing comprehensive epitope-based vaccines and diagnostic kits against Hendra virus after further in-vivo studies.
\end{abstract}

Keywords Hendra virus $\cdot$ T-cell epitope $\cdot$ Antigenicity $\cdot$ HLA alleles, Modeling $\cdot$ Population coverage $\cdot$ Vaccine designing

\section{Introduction}

Hendra virus $(\mathrm{HeV})$ initially emerged in an outbreak of acute respiratory disease amongst horses and humans in Australia, 1994 (Murray et al. 1995). Patients of these cases were found to have infected with close contact to diseased horses. Initial cases of Hendra infection clinically diagnosed as severe respiratory infection (Selvey et al. 1995; Hanna et al. 2006; O'Sullivan et al. 1997; Playford et al. 2010). There is no

\section{K. Sharma}

sharmadr_dinesh@yahoo.in

Jiwaji University, Gwalior, Madhya Pradesh, India

2 Mangalayatan University, Aligarh, Uttar Pradesh, India

3 Veer Narmad South Gujarat University, Surat, Gujarat, India

4 Dept of Microbiology, College of Life Science, Cancer Hospital Campus Gwalior, Gwalior, Madhya Pradesh, India specific treatment for human cases of Hendra virus. Flying foxes (pteopid bats) considered as the natural reservoir of $\mathrm{HeV}$. Transmission of viral infection to humans occurred likely due to exposure of mucous membrane or non-intact skin to nasal secretions, urine and blood of infected horses (Mire et al. 2015). HeV was primarily isolated from infected uterine fluid and fetal tissue of bat species Pteropus policephalus and Pteropus alecto respectively (Halpin et al. 2000). Equivac Hev, a subunit vaccine from glycoprotein of $\mathrm{HeV}$ is the only approved licensed vaccine from Australian government which is used for prevention of $\mathrm{Hev}$ in the horse population.

Peptide vaccines are considered an alternative to classical vaccines that are trying to address issues of possible vaccine side effects related to vaccination with a heterogeneous multicomponent preparation. The peptide-based vaccines include chemical approach to synthesize the selected epitopes that are specific and trigger immune responses. 
Table 1 Template PDB ID for modeling of selected HLA class II alleles

\begin{tabular}{lll}
\hline S. no. & HLA class II allele & $\begin{array}{l}\text { PDB ID } \\
\text { of tem- } \\
\text { plate }\end{array}$ \\
\hline 1 & DRB $1 * 0817$ & $1 \mathrm{~A} 6 \mathrm{~A}$ \\
2 & DRB $1 * 1101$ & $1 \mathrm{YMM}$ \\
3 & DRB $1 * 0405$ & $2 \mathrm{SEB}$ \\
4 & DRB $1 * 0410$ & $2 \mathrm{SEB}$ \\
5 & DRB $1 * 1104$ & $2 \mathrm{WBJ}$ \\
6 & DRB $1 * 0301$ & $1 \mathrm{~A} 6 \mathrm{~A}$ \\
7 & DRB $1 * 1301$ & $2 \mathrm{WBJ}$ \\
8 & DRB $1 * 0701$ & $1 \mathrm{AQD}$ \\
9 & $\mathrm{DRB} 1 * 1102$ & $2 \mathrm{WBJ}$ \\
10 & DRB $1 * 1128$ & $1 \mathrm{~A} 6 \mathrm{~A}$ \\
11 & DRB $1 * 0402$ & $4 \mathrm{MDI}$ \\
12 & DRB $1 * 1304$ & $1 \mathrm{YMM}$ \\
13 & DRB $1 * 0804$ & $2 \mathrm{SEB}$ \\
14 & DRB $1 * 0806$ & $2 \mathrm{WBJ}$ \\
\hline
\end{tabular}

Immunoinformatic focuses mainly on prediction of potential epitopes which brings down the laboratory analysis cost $\&$ time of potential vaccine candidate. Using immunoinformatic tools, immunologist can screen and analyze short sequences from the full-length foreign proteins which can act as an immunogenic epitopes \& facilitating to be a vaccine candidate (Li et al. 2014; Kamthania and Sharma 2016). Important implication of this study is to screen promiscuous T-cell epitopes from $\mathrm{HeV}$ proteins viz matrix, glycoprotein, nucleocapsid, fusion, $\mathrm{C}$ protein, $\mathrm{V}$ protein, $\mathrm{W}$ protein, and polymerase. These predicted promiscuous $\mathrm{T}$-cell epitopes may be the promising targets for epitope-based vaccine design for $\mathrm{HeV}$.

\section{Materials and Methods}

\section{Retrieval of Amino Acid Sequence}

The amino acid sequences of matrix, glycoprotein, nucleocapsid, fusion, $\mathrm{C}$ protein, $\mathrm{V}$ protein, $\mathrm{W}$ protein and polymerase, were retrieved from NCBI protein sequence database (http://www.ncbi.nlm.nih.gov/protein). The total of 22 nucleocapsid, 20 matrix, 22 fusion, 22 glycoprotein, $14 \mathrm{~W}$ protein, $17 \mathrm{~V}$ protein, $15 \mathrm{C}$ protein and 15 polymerase protein sequences from different $\mathrm{HeV}$ strains, available at NCBI database were retrieved \& downloaded in FASTA format.

\section{Antigenicity Prediction}

VaxiJen server (http://www.ddg-pharmfac.net/vaxijen/VaxiJ en/VaxiJen.html) (Doytchinova and Flower 2007) was used with default parameters to predict the antigenicity of $\mathrm{HeV}$ candidate proteins. The threshold value of vaxiJen was set to 0.4 (default for viruses). The viral proteins having Vaxijen score above 0.4 values were considered to be antigenic in nature. Antigenic proteins were chosen for further study.

\section{Promiscuous T-Cell Epitope Prediction}

The prediction of promiscuous T-cell epitope binder to HLA class-II alleles was performed by ProPred (Singh and Raghava 2001). ProPred is an on-line web tool; it utilizes matrix-based prediction of HLA (Class II alleles) binding sites in an antigenic protein sequence.

\section{D Structure Prediction of Promiscuous T-cell Epitopes}

The PEPstrMOD (Singh et al. 2015) method performed to find out the tertiary structure of selected small epitope with

Table 2 IC $_{50}$ values of alleles with their corresponding predicted potential T-cell epitopes IRIFVPATN, MRNLLSQSL, VRRAGKYYS and VRLKCLLCG with an affinity of $<500 \mathrm{nM}$

\begin{tabular}{|c|c|c|c|}
\hline T-cell epitope & $\begin{array}{l}\text { Total no. of } \\
\text { HLA-peptide } \\
\text { binders }\end{array}$ & MHC-I alleles with $\mathrm{IC}_{50}$ values (bracketed) & MHC-II alleles with $\mathrm{IC}_{50}$ values (bracketed) \\
\hline IRIFVPATN & 6 & $\begin{array}{l}\text { HLA-A*02:03(16.00), HLA-A*02:06(439.54), HLA- } \\
\text { A*03:01(334.20),HLA-A*11:01(34.04), HLA- } \\
\text { A*68:01(59.16), HLA-A*68:02(192.75) }\end{array}$ & - \\
\hline MRNLLSQSL & 9 & $\begin{array}{l}\text { HLA-A*02:03(11.72), HLA-A*02:06(510.50), } \\
\text { HLA-A*03:01(340.41), HLA-A*11:01(31.84), } \\
\text { HLA-A*31:01(411.15), HLA-A*68:01(180.72), } \\
\text { HLA-B*35:01(408.32) }\end{array}$ & HLA-DRB*01:01(35.97), HLA-DRB*07:01(428.55) \\
\hline VRRAGKYYS & 7 & $\begin{array}{l}\text { HLA-A*02:02(179.47), HLA-A*02:06(193.20), } \\
\text { HLA-A*11:01(75.68), HLA-A*68:01(457.09) }\end{array}$ & $\begin{array}{l}\text { HLA-DRB*01:01(381.94), HLA- } \\
\text { DRB*04:01(210.38), HLA-DRB*07:01(297.17) }\end{array}$ \\
\hline VRLKCLLCG & 6 & $\begin{array}{l}\text { HLA-A*02:01(112.46), HLA-A*02:03(151.01), } \\
\text { HLA-A*02:06(57.94), HLA-A*11:01(123.88), } \\
\text { HLA-A*68:01(382.82) }\end{array}$ & HLA-DRB*04:01(51.29) \\
\hline
\end{tabular}


Table 3 Conservancy analysis using Conservation across antigen tool of IEDB revealed that all the four chosen epitopes are $100 \%$ conserved across all the $\mathrm{HeV}$ strain's respective protein sequences retrieved from NCBI database

\begin{tabular}{llllcl}
\hline S. no. & Protein name & $\begin{array}{l}\text { Amino acid sequence } \\
\text { of selected epitope }\end{array}$ & $\begin{array}{l}\text { Selected } \\
\text { epitope } \\
\text { length }\end{array}$ & Position & $\begin{array}{l}\text { Percent of protein sequence } \\
\text { match at identity } \geq 100 \%\end{array}$ \\
\hline 1 & Nucleocapsid & IRIFVPATN & 9 & $35-43$ & $100.00 \%(22 / 22)$ \\
2 & Nucleocapsid & MRNLLSQSL & 9 & $217-225$ & $100.00 \%(22 / 22)$ \\
3 & Matrix & VRRAGKYYS & 9 & $243-251$ & $100.00 \%(20 / 20)$ \\
4 & Fusion & VRLKCLLCG & 9 & $6-14$ & $100.00 \%(22 / 22)$ \\
\hline
\end{tabular}

Table 4 VaxiJen Results of antigenicity

\begin{tabular}{ll}
\hline Protein & Overall antigen prediction \\
\hline Nucleocapsid & 0.5385 (Probable ANTIGEN) \\
Matrix & 0.4422 (Probable ANTIGEN) \\
Fusion & 0.5529 (Probable ANTIGEN) \\
Glycoprotein & 0.5380 (Probable ANTIGEN) \\
W protein & 0.4747 (Probable ANTIGEN) \\
nonstructural protein V & 0.4978 (Probable ANTIGEN) \\
nonstructural protein C & 0.3540 (Probable NON-ANTIGEN) \\
Polymerase & 0.4843 (Probable ANTIGEN) \\
\hline
\end{tabular}

sequence length of nine residues. The PEPstrMOD tool prediction strategy utilizes the secondary structure data anticipated by PSIPRED (Kaur and Raghava 2004) and $\beta$-turns data anticipated by BetaTurns (Jones et al. 1999).

\section{Modeling of HLA Class II Alleles}

The sequences of alleles viz DRB $1 * 1304$, DRB $1 * 0804$, DRB $1 * 0405$, DRB1*0806, DRB1*0402, DRB1*0701, DRB $1 * 1301$, DRB $1 * 1104$, DRB $1 * 1102$. DRB $1 * 0410$, DRB1*1128, DRB1*1101, DRB1*0301, \& DRB1*0817 were downloaded from IMGT/HLA database (http://www. ebi.ac.uk/ipd/imgt/hla/allele.html) (Robinson et al. 2012). BLASTP program (Altschul et al. 1990) was used to find the templates for these allele sequences and respective PDB IDs (Table 1) retrieved from Protein Data Bank (http://www. rcsb.org/pdb). MODELLER 9.17 (Šali et al. 1995) was used for homology modeling of HLA alleles. Five models were generated by modeller software and the best model chosen based on their lowest predicted discrete optimized protein energy (DOPE) score. The modeled alleles overall quality was determined using Ramachandran plot analysis utilizing PROCHECK (Laskowski et al. 1993), which detect the geometrical orientation of each amino acid residues with respect to stereochemical parameters.

\section{Molecular Docking}

AutoDock 4.2 (Morris et al. 1998) was utilized to form the docking complex of predicted T-cell epitopes and HLA alleles. In case of rigid protein \& flexible ligand during autodock, the Lamarckian Genetic Algorithm (LGA) was used. The best conformation of docked complex was chosen on the basis of minimum binding energy and best fitting of epitopeHLA allele complex with highest number of H-bonds formation. Python Molecular Viewer (Sanner et al. 1999) used to visualize the docked complex of HLA allele with predicted $\mathrm{T}$ cell epitope.

\section{Docked Complex Stability Validation by MD Simulation}

NAMD used for MD simulation of selected docked complex (Phillips et al. 2005). VMD analyses \& view the results of MD simulation \& also interface with NAMD (Humphrey et al. 1996). Protein structure file (psf) was made using the topology files and initial pdb files of the HLA II alleleepitope docked complex utilizing psfgen package of VMD. NAMD created the trajectory DCD file. The result of simulation was analyzed by calculation the Root mean square deviation (RMSD) of the docked complex. The rmsd.dat file contains the value of RMSD which was further analyzed by Microsoft office excel.

\section{Toxicity Prediction of the Selected T-Cell Epitopes}

ToxinPred (http://crdd.osdd.net/raghava/toxinpred/) (Gupta et al. 2013) was utilized to calculate toxicity of predicted T- cell epitopes. ToxinPred is an in-silico method to predict toxic/non-toxic peptides. ToxinPred was run with default parameters and only non-toxic T-cell epitopes were selected for further study.

\section{HLA-Distribution Analysis}

MHCPred (http://www.ddg-pharmfac.net/mhcpred/MHCPr ed/) was utilized to select the high affinity HLA class II binder for selected epitopes with their $\mathrm{IC}_{50}$ (half maximal inhibitory concentration) value. The alleles having cut-off value of $\mathrm{IC}_{50}$ between 0.01 and $500 \mathrm{nM}$ were selected. 
Table 5 ProPred predicted T-cell epitope for HLA Class II with binding scores

\begin{tabular}{|c|c|c|c|c|c|c|}
\hline Protein name & Accession no. (NCBI) & $\begin{array}{l}\text { Amino acid } \\
\text { length }\end{array}$ & Start position & Epitopes & HLA class II alleles & $\begin{array}{l}\text { Propred (\% of } \\
\text { highest score) }\end{array}$ \\
\hline \multirow{8}{*}{ Nucleocapsid } & \multirow[t]{8}{*}{ NP_047106 } & \multirow[t]{8}{*}{532} & \multirow[t]{4}{*}{128} & \multirow[t]{4}{*}{ MRILKTARE } & DRB $1 * 0410$ & 84.04 \\
\hline & & & & & DRB1*0806 & 75.58 \\
\hline & & & & & DRB $1 * 0405$ & 73.4 \\
\hline & & & & & DRB1*1304 & 68.89 \\
\hline & & & \multirow[t]{2}{*}{34} & \multirow[t]{2}{*}{ IRIFVPATN } & DRB $1 * 1304$ & 62.22 \\
\hline & & & & & $\mathrm{DRB} 1 * 0806$ & 61.63 \\
\hline & & & 476 & LNLRSRLAA & DRB $1 * 0817$ & 55.45 \\
\hline & & & 216 & MRNLLSQSL & DRB1*0701 & 51.72 \\
\hline \multirow[t]{5}{*}{ Matrix } & \multirow[t]{5}{*}{ NP_047110 } & \multirow[t]{5}{*}{352} & 242 & VRRAGKYYS & DRB1*1301 & 57.95 \\
\hline & & & & & $\mathrm{DRB} 1 * 0301$ & 55.26 \\
\hline & & & 261 & MKLQFSLGS & DRB $1 * 0421$ & 52.22 \\
\hline & & & 277 & IKINGVISK & DRB1*1104 & 51.81 \\
\hline & & & 195 & FRRNNAIAF & DRB $1 * 0701$ & 54.31 \\
\hline \multirow[t]{4}{*}{ Fusion } & \multirow[t]{4}{*}{ NP_047111 } & \multirow[t]{4}{*}{546} & 493 & MIILYVLSI & DRB1*1104 & 73.49 \\
\hline & & & 315 & VLIRNTLIS & DRB $1 * 0402$ & 73.96 \\
\hline & & & 5 & VRLKCLLCG & $\mathrm{DRB} 1 * 0806$ & 66.28 \\
\hline & & & 314 & FVLIRNTLI & DRB1*0701 & 68.1 \\
\hline \multirow[t]{3}{*}{ Glycoprotein } & \multirow[t]{3}{*}{ NP_047112 } & \multirow[t]{3}{*}{604} & 316 & IRLAVRPKS & $\mathrm{DRB} 1 * 0301$ & 63.16 \\
\hline & & & 152 & LKIHECNIS & DRB1*1301 & 60.23 \\
\hline & & & 474 & LRVQWRNNS & DRB1*1102 & 60.71 \\
\hline $\mathrm{W}$ protein & YP_007188593 & 447 & 355 & LRLREPPQS & DRB $1 * 0806$ & 63.95 \\
\hline \multirow[t]{5}{*}{$\mathrm{V}$ protein } & \multirow[t]{5}{*}{ NP_047108 } & \multirow[t]{5}{*}{457} & \multirow[t]{2}{*}{355} & \multirow[t]{2}{*}{ LRLREPPQS } & DRB $1 * 0804$ & 68.75 \\
\hline & & & & & DRB $1 * 0806$ & 63.95 \\
\hline & & & \multirow[t]{3}{*}{304} & \multirow[t]{3}{*}{ VRRKDSLMQ } & DRB $1 * 0806$ & 70.93 \\
\hline & & & & & DRB $1 * 0817$ & 68.32 \\
\hline & & & & & DRB $1 * 0804$ & 66.25 \\
\hline \multirow[t]{5}{*}{ Polymerase } & \multirow[t]{5}{*}{ NP_047113 } & \multirow[t]{5}{*}{2244} & \multirow[t]{3}{*}{1169} & \multirow[t]{3}{*}{ FLILNRLLS } & DRB1*1305 & 82.76 \\
\hline & & & & & DRB1*1128 & 82.76 \\
\hline & & & & & DRB $1 * 1101$ & 81.93 \\
\hline & & & \multirow[t]{2}{*}{468} & \multirow[t]{2}{*}{ MYMKDKALS } & DRB $1 * 0804$ & 82.5 \\
\hline & & & & & DRB $1 * 0806$ & 76.74 \\
\hline
\end{tabular}

\section{Population Coverage Analysis}

IEDB (Immune Epitope Database and Analysis Resource) population coverage tool (http://tools.immuneepitope.org/ tools/population/iedb_input) (Bui et al. 2006) was used to study the worldwide geographical population coverage by the selected epitopes and HLA Class II alleles pair as resulted from MHCPred (Table 2). Default parameters were set while running the tool. IEDB conservancy tool consist data set frequencies of 3245 alleles for 115 countries, 21 ethnicities and 16 geographical areas. The prediction was taken utilizing the most resent information to set from Allele Frequency Net Database (Gonzalez-Galarza et al. 2010) at IEDB.

\section{Conservancy Analysis of Selected Epitopes}

To find out the degree of conservation, all the selected epitopes were aligned against all respective source protein sequences retrieved from NCBI database using EBI-clustal Omega program (Sievers et al. 2011). The Multiple sequence alignment (MSA) was visualized using Jalview (Waterhouse et al. 2009). Conservancy analysis was again performed for the selected T-cell epitopes by conservancy tool of IEDB (Table 3) (Bui et al. 2007). 
Table 6 Toxicity prediction of the peptides by ToxinPred

\begin{tabular}{|c|c|c|c|c|c|c|c|}
\hline Peptide sequence & SVM Score & Prediction & Hydroph-obicity & Hydropathicity & Hydrophilicity & Charge & Mol. wt \\
\hline MRILKTARE & -1.61 & Non-toxin & -0.41 & -0.57 & 0.69 & 2.00 & 1117.50 \\
\hline IRIFVPATN & -1.23 & Non-toxin & 0.02 & 0.83 & -0.59 & 1.00 & 1030.36 \\
\hline LNLRSRLAA & -1.09 & Non-toxin & -0.26 & 0.19 & 0.01 & 2.00 & 1013.33 \\
\hline MRNLLSQSL & -1.32 & Non-toxin & -0.20 & 0.02 & -0.30 & 1.00 & 1061.40 \\
\hline VRRAGKYYS & -0.95 & Non-toxin & -0.43 & -1.19 & 0.30 & 3.00 & 1099.37 \\
\hline MKLQFSLGS & -0.98 & Non-toxin & -0.02 & 0.32 & -0.40 & 1.00 & 1010.35 \\
\hline IKINGVISK & -1.02 & Non-toxin & -0.02 & 0.58 & -0.04 & 2.00 & 0971.35 \\
\hline FRRNNAIAF & -0.97 & Non-toxin & -0.26 & -0.26 & -0.16 & 2.00 & 1108.38 \\
\hline MIILYVLSI & -0.99 & Non-toxin & 0.42 & 2.79 & -1.53 & 0.00 & 1064.54 \\
\hline VLIRNTLIS & -0.77 & Non-toxin & 0.02 & 1.26 & -0.62 & 1.00 & 1028.40 \\
\hline VRLKCLLCG & -0.69 & Non-toxin & -0.05 & 1.31 & -0.32 & 2.00 & 1004.45 \\
\hline FVLIRNTLI & -0.74 & Non-toxin & 0.12 & 1.66 & -0.93 & 1.00 & 1088.50 \\
\hline IRLAVRPKS & -1.22 & Non-toxin & -0.32 & -0.11 & 0.41 & 3.00 & 1039.41 \\
\hline LKIHECNIS & -0.64 & Non-toxin & -0.11 & 0.04 & -0.04 & 0.50 & 1056.38 \\
\hline LRVQWRNNS & -0.61 & Non-toxin & -0.48 & -1.47 & 0.02 & 2.00 & 1172.44 \\
\hline LRLREPPQS & -0.72 & Non-toxin & -0.46 & -1.38 & 0.66 & 1.00 & 1095.39 \\
\hline LRLREPPQS & -0.72 & Non-toxin & -0.46 & -1.38 & 0.66 & 1.00 & 1095.39 \\
\hline VRRKDSLMQ & -1.26 & Non-toxin & -0.55 & -1.20 & 0.88 & 2.00 & 1132.47 \\
\hline LRMMEVLKE & -1.47 & Non-toxin & -0.22 & 0.02 & 0.48 & 0.00 & 1148.58 \\
\hline LQFLKKLGK & -0.70 & Non-toxin & -0.18 & -0.16 & 0.14 & 3.00 & 1074.52 \\
\hline FLILNRLLS & -1.28 & Non-toxin & 0.09 & 1.52 & -0.89 & 1.00 & 1088.50 \\
\hline MYMKDKALS & -0.96 & Non-toxin & -0.21 & -0.44 & 0.23 & 1.00 & 1086.45 \\
\hline
\end{tabular}

\section{Results and Discussion}

\section{Antigenicity Prediction}

Amino acid sequences of matrix, glycoprotein, nucleocapsid, fusion, $\mathrm{C}$ protein, $\mathrm{V}$ protein, $\mathrm{W}$ protein and polymerase proteins were screened by VaxiJen. All the proteins were found antigenic except one nonstructural protein $\mathrm{C}$ which is nonantigenic at threshold value 0.4 (default threshold for viral proteins) (Table 4). Fusion protein had been predicted as highest antigenic with score of 0.5529 among all candidate proteins.

\section{Promiscuous T-cell Epitope selection and analysis}

Antigenic $\mathrm{HeV}$ proteins were subjected to Propred for selection of HLA Class II specific T- cell epitopes binders. Epitopes showing highest score with the maximum number of HLA Class II alleles binders were selected at a threshold value of $4 \%$ (Table 5).

\section{Toxicity Prediction of the Peptide Epitopes}

ToxinPred (Gupta et al. 2013) used for toxicity prediction of selected T- cell epitopes. ToxinPred tool is a unique in-silico method based on Support Vector Machine (SVM) in predicting toxicity of peptides along with important physico-chemical properties viz hydropathicity, hydrophilicity, hydrophobicity, charge and molecular weight. The selected epitopes were subjected to ToxinPred and only non-toxic T-cell epitopes were selected for further studies (Table 6).

\section{Structure Modeling of T-cell Epitopes and Alleles}

3D structures of selected epitopes were predicted by PEPstrMOD. Modeller 9.17 was employed to generate homology model of alleles: DRB1*1304, DRB1*0804, DRB $1 * 0405$, DRB $1 * 0806$, DRB $1 * 0402$, DRB $1 * 0701$, DRB1*1301, DRB1*1104, DRB1*1102. DRB1*0410, DRB1*1128, DRB1*1101, DRB1*0301, \& DRB1*0817; template used (PDB ID): 1YMM, 2SEB, 2SEB, 2WBJ, 4MDI, 1AQD, 2WBJ, 2WBJ, 2WBJ, 2SEB, 1A6A, $1 \mathrm{YMM}, 1 \mathrm{~A} 6 \mathrm{~A} \&$ \& $1 \mathrm{~A} 6 \mathrm{~A}$ respectively (Table 1). PROCHECK was utilized to analyze selected models quality. Ramachandran result of HLA alleles model (DRB1*0701, DRB $1 * 0301$,DRB $1 * 1304$ and DRB $1 * 0806$ alleles) forming best HLA allele-epitope complex, based on docking study (Table 7), is shown in Fig. 1 a-d. 
Table 7 Binding energy determination by autodock

\begin{tabular}{|c|c|c|c|c|c|c|c|c|}
\hline Protein name & Epitope & HLA Class II allele & Binding energy & $\begin{array}{l}\text { Intermo- } \\
\text { lecular } \\
\text { energy }\end{array}$ & Internal energy & Torsional energy & $\begin{array}{l}\text { Vdw- } \\
\text { lbDesolv } \\
\text { energy }\end{array}$ & $\begin{array}{l}\text { Electro- } \\
\text { static } \\
\text { energy }\end{array}$ \\
\hline \multirow[t]{8}{*}{ Nucleocapsid } & \multirow[t]{4}{*}{ MRILKTARE } & DRB1_0410 & 4.01 & -7.93 & -8.58 & 11.93 & -6.28 & -1.65 \\
\hline & & DRB1_0806 & 2.66 & -9.28 & -7.49 & 11.93 & -6.95 & -2.33 \\
\hline & & DRB1_0405 & 2.28 & -9.66 & -7.2 & 11.93 & -9.5 & -0.16 \\
\hline & & DRB1_1304 & 3.91 & -8.02 & -7.12 & 11.93 & -7.44 & -0.58 \\
\hline & \multirow[t]{2}{*}{ IRIFVPATN } & DRB1_1304 & -2.56 & -11.51 & -7.83 & 8.95 & -11.25 & -0.26 \\
\hline & & DRB1_0806 & -1.88 & -10.82 & -6.55 & 8.95 & -10.92 & -0.1 \\
\hline & LNLRSRLAA & DRB1_0817 & 0.13 & -10.01 & -6.01 & 10.14 & -9.43 & -0.59 \\
\hline & MRNLLSQSL & DRB1_0701 & -2.76 & -13.8 & -6.82 & 11.04 & -13.86 & -0.06 \\
\hline \multirow[t]{5}{*}{ Matrix } & \multirow[t]{2}{*}{ VRRAGKYYS } & DRB1_1301 & 1.12 & -1.32 & -7.87 & 10.44 & -9.030 & -0.29 \\
\hline & & DRB1_0301 & -1.01 & -11.45 & -7.88 & 10.44 & -11.38 & -0.07 \\
\hline & MKLQFSLGS & DRB1_0421 & -0.15 & -10.59 & -6.86 & 10.44 & -10.02 & -0.57 \\
\hline & IKINGVISK & DRB1_1104 & -0.62 & -11.06 & -5.7 & 10.44 & -9.79 & -1.28 \\
\hline & FRRNNAIAF & DRB1_0701 & -0.65 & -11.09 & -9.0 & 10.44 & -11.07 & -0.01 \\
\hline \multirow[t]{4}{*}{ Fusion } & MIILYVLSI & DRB1_1104 & 1.06 & -9.08 & -10.05 & 10.14 & -8.78 & -0.3 \\
\hline & VLIRNTLIS & DRB1_0402 & -0.24 & -10.38 & -8.75 & 10.14 & -10.31 & -0.07 \\
\hline & VRLKCLLCG & DRB1_0806 & -1.51 & -11.66 & -8.25 & 10.14 & -11.39 & -0.26 \\
\hline & FVLIRNTLI & DRB1_0701 & -0.73 & -11.17 & -7.76 & 10.44 & -11.31 & -0.14 \\
\hline \multirow[t]{3}{*}{ Glycoprotein } & IRLAVRPKS & DRB1_0301 & -0.22 & -10.37 & -6.58 & 10.14 & -10.05 & -0.32 \\
\hline & LKIHECNIS & DRB1_1301 & 3.76 & -6.98 & -10.91 & 10.74 & -6.68 & -0.3 \\
\hline & LRVQWRNNS & DRB1_1102 & 1.53 & -10.1 & -8.87 & 11.63 & -9.72 & -0.38 \\
\hline $\mathrm{W}$ protein & LRLREPPQS & DRB1_0806 & -0.38 & 10.53 & -7.13 & 10.14 & -10.64 & -0.11 \\
\hline \multirow[t]{5}{*}{$\mathrm{V}$ protein } & \multirow[t]{2}{*}{ LRLREPPQS } & DRB1_0804 & -0.76 & -10.9 & -9.86 & 10.14 & -11.36 & -0.46 \\
\hline & & DRB1_0806 & 0.94 & -9.2 & -7.63 & 10.14 & -9.33 & -0.13 \\
\hline & \multirow[t]{3}{*}{ VRRKDSLMQ } & DRB1_0806 & 2.87 & -9.36 & -8.99 & 12.23 & -8.98 & -0.38 \\
\hline & & DRB1_0817 & 4.28 & -7.98 & -8.49 & 12.23 & -8.04 & -0.06 \\
\hline & & DRB1_0804 & 1.98 & -10.25 & -7.44 & 12.23 & -10.35 & -0.1 \\
\hline \multirow[t]{5}{*}{ Polymerase } & \multirow[t]{3}{*}{ FLILNRLLS } & DRB1_1305 & 0.29 & -10.45 & -6.52 & 10.74 & -10.02 & -0.43 \\
\hline & & DRB1_1128 & -0.55 & -11.29 & -7.46 & 10.74 & -4.62 & -0.16 \\
\hline & & DRB1_1101 & 0.9 & -9.84 & -8.61 & 10.74 & -9.77 & -0.07 \\
\hline & \multirow[t]{2}{*}{ MYMKDKALS } & DRB1_0804 & 2.38 & -8.96 & -7.89 & 11.34 & -6.71 & -2.25 \\
\hline & & DRB1_0806 & 2.13 & -9.2 & -9.37 & 11.34 & -7.03 & -2.18 \\
\hline
\end{tabular}

\section{Binding Energy Determination of Epitope \& HLA Class II Allele}

The interaction studies of selected T-cell epitopes with their respective highest ProPred scorer HLA class II allele binders were predicted by utilizing Autodock 4.2 (Table 7). Amongst the docked complexes, nucleocapsid protein peptide IRIFVPATN were dock with two alleles DRB $1 * 0806 \&$ DRB $1 * 1304$ and nucleocapsid protein peptide MRNLLSQSL with DRB $1 * 0701$ allele formed stable complexes having binding energy value of $-1.88 \mathrm{kcal} /$ $\mathrm{mol},-2.56 \mathrm{kcal} / \mathrm{mol}$ and $-2.76 \mathrm{kcal} / \mathrm{mol}$ respectively. Matrix protein peptide VRRAGKYYS was docked with DRB $1 * 0301$ HLA allele, it showed binding energy value of $-1.01 \mathrm{kcal} / \mathrm{mol}$. Fusion protein peptide VRLKCLLCG was docked with DRB1*0806 HLA allele and has shown binding energy value of $-1.51 \mathrm{kcal} / \mathrm{mol}$ Figs. 2, 3, 4, 5 and 6 respectively. The docked complexes were visualized by Python molecular viewer.

\section{Docked Complex Stability Validation \& RMSD Plot}

The lowest binding energy docked complexes of T-cell epitope \& HLA Class II allele were subjected to MD simulation by NAMD. The RMSD plot of docked complexes IRIFVPATN-DRB $1 * 0806$, IRIFVPATN-DRB $1 * 1304$, MRNLLSQSL-DRB $1 * 0701$, VRRAGKYYS-DRB $1 * 0301$ and VRLKCLLCG-DRB $1 * 0806$ showed the highest peak at $0.95 \AA, 0.98 \AA, 0.99 \AA, 1.02 \AA$ and $1.01 \AA$ RMSD (Fig. 7 a-c, 8,9$)$ respectively. 
(a)

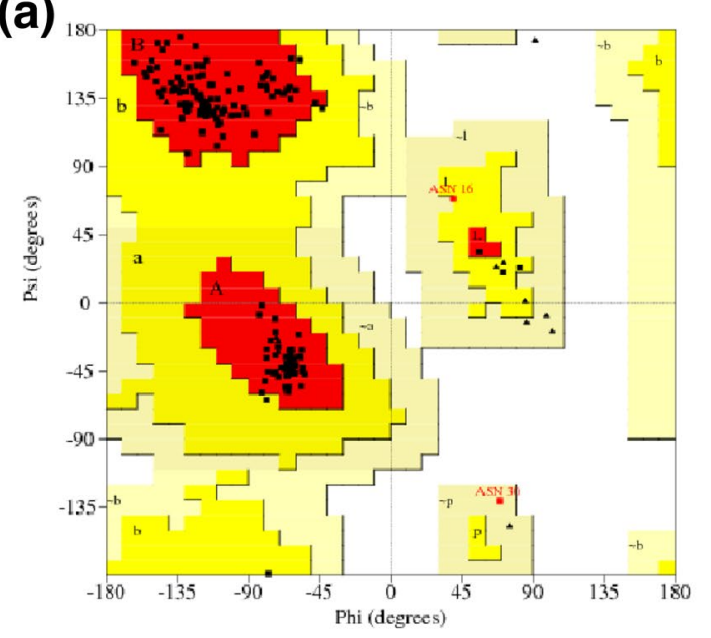

(c)

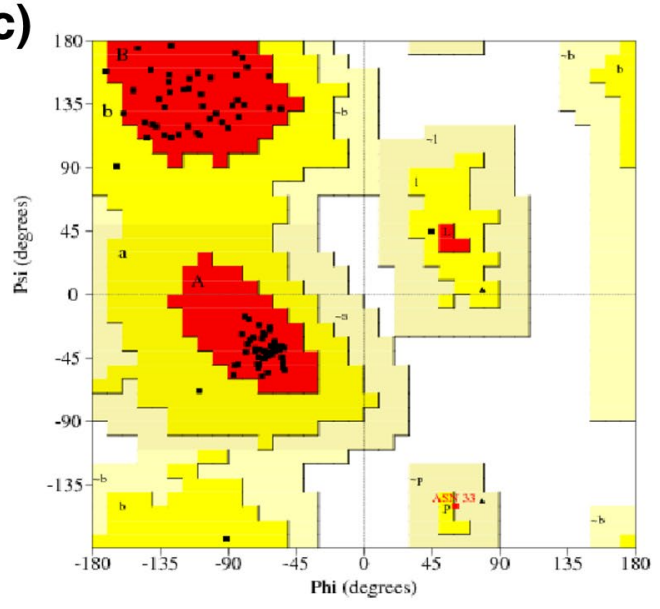

(b)

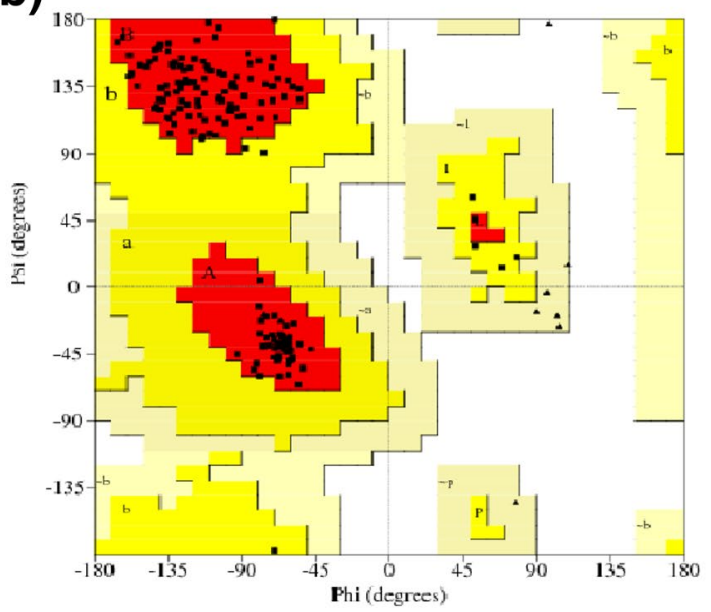

(d)

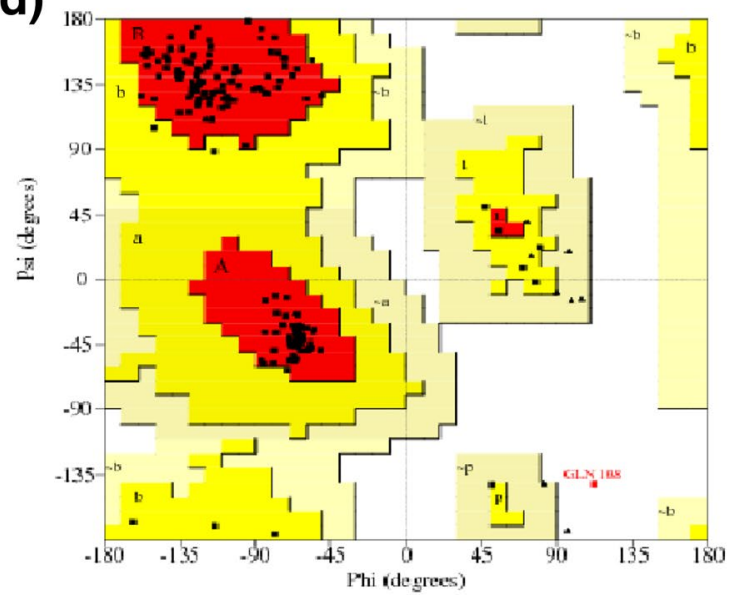

Fig. 1 Ramachandran plot of protein model: a DRB1*0701, b DRB1*0301, c DRB1*1304, d DRB1*0806

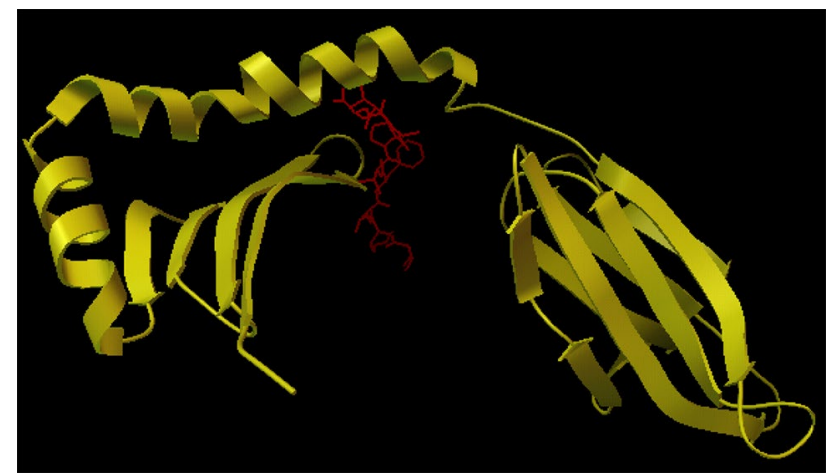

Fig. 2 Docked complex of Nucleocapsid protein peptide IRIFVPATN with DRB1*0806 allele

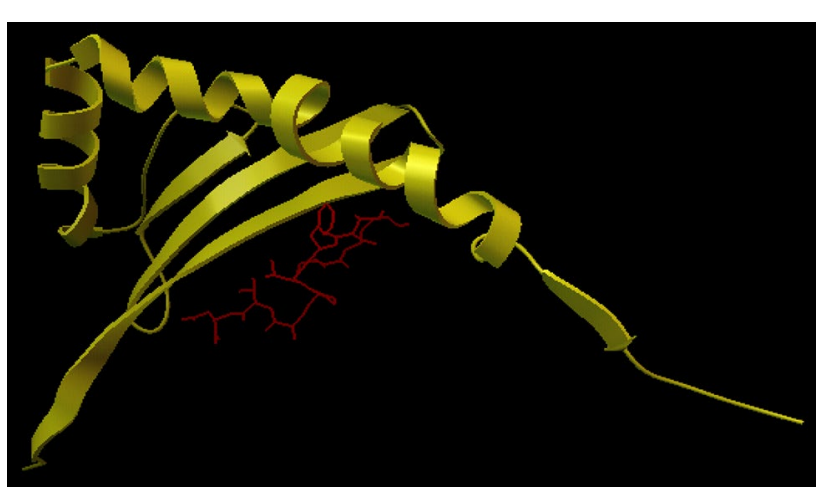

Fig. 3 Docked complex of Nucleocapsid protein peptide IRIFVPATN with DRB1*1304 allele 
Fig. 4 Docked complex of Nucleocapsid protein peptide MRNLLSQSL and DRB $1 * 0701$ allele Formation of one H-bond with SER123 (OG) and peptide $\operatorname{MET} 1(\mathrm{~N})$

Fig. 5 Docked complex of Matrix protein peptide VRRAGKYYS and $\mathrm{DRB} 1 * 0301$ allele. Formation of one H-bond with PHE13 (O) and peptide SER9 $(\mathrm{N})$
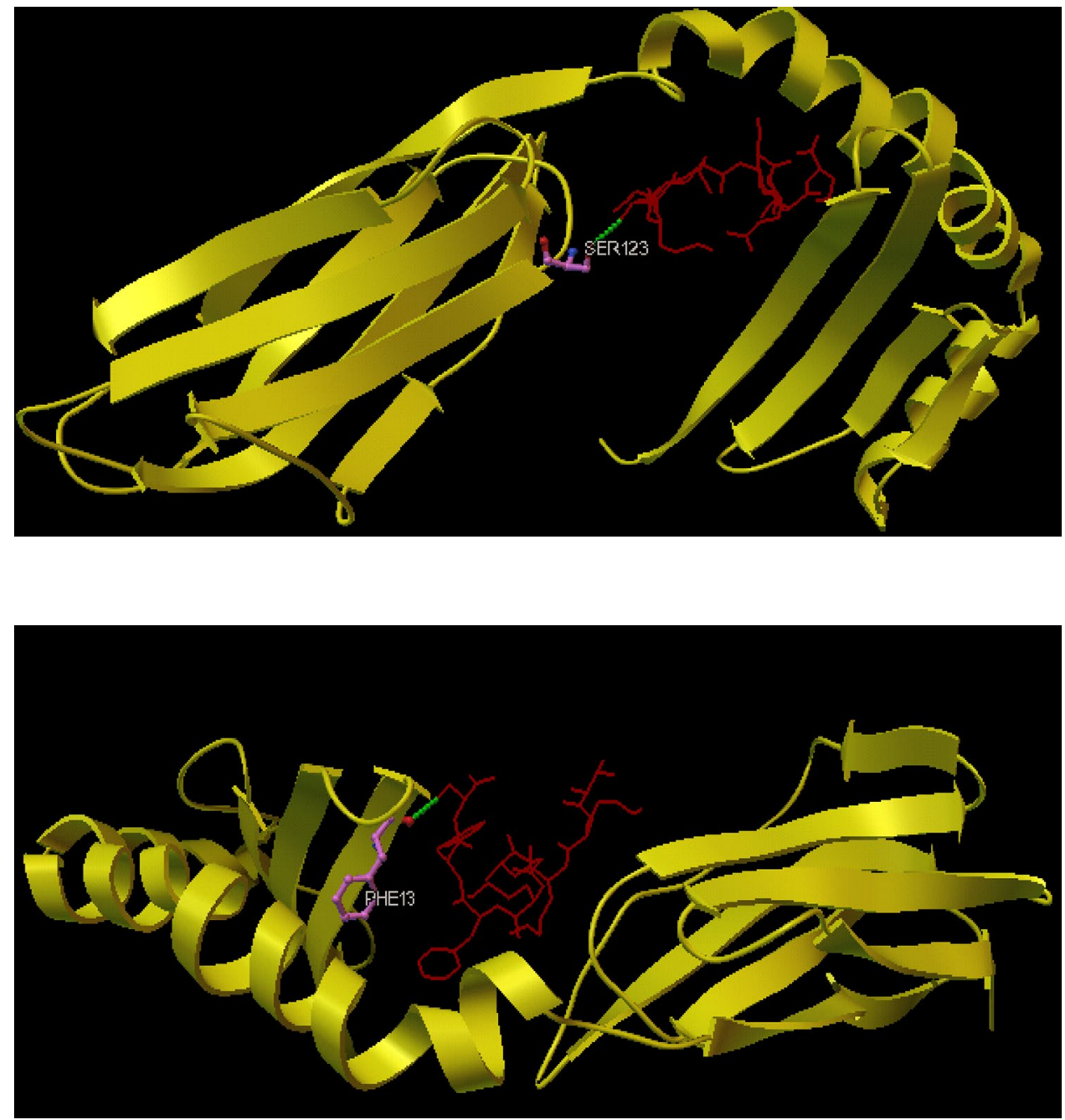

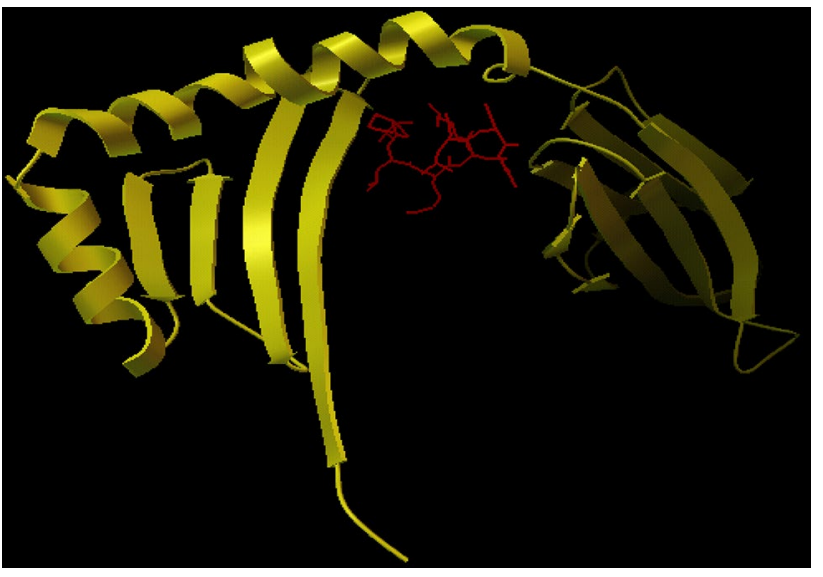

Fig. 6 Docked complex of Fusion protein peptide VRLKCLLCG with DRB $1 * 0806$ allele

\section{Population Coverage Estimation of Predicted T-Cell Epitopes}

MHCPred (Guan et al. 2003) was employed to predict the potential HLA allele binders for selected $\mathrm{T}$ cell epitopes IRIFVPATN, MRNLLSQSL, VRRAGKYYS and VRLKCLLCG, along with their interacting affinity with $\mathrm{IC}_{50}$ value to be $<=500 \mathrm{nM}$ (Table 2). IEDB tool predicted the four epitopes IRIFVPATN, MRNLLSQSL, VRRAGKYYS and VRLKCLLCG have population coverage of $40.24 \%$, $56.78 \%, 36.82 \%$ and $57.08 \%$ respectively for total world population (Table 8 ). Highest coverage of $59.74 \%, 53.24 \%$ and $66.78 \%$ for epitope IRIFVPATN, VRRAGKYYS and VRLKCLLCG respectively were found for population of the Northeast Asia. Epitope MRNLLSQSL has highest coverage of $70.28 \%$ in the population of South Asia. The results of high population coverage suggested that the putative T-cell epitopes cover vast majority of geographic population. 
(a)

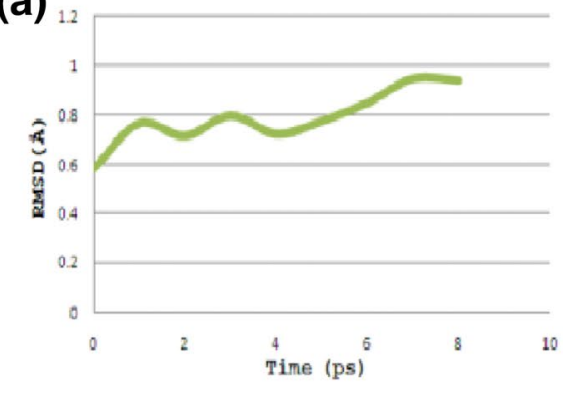

(b)

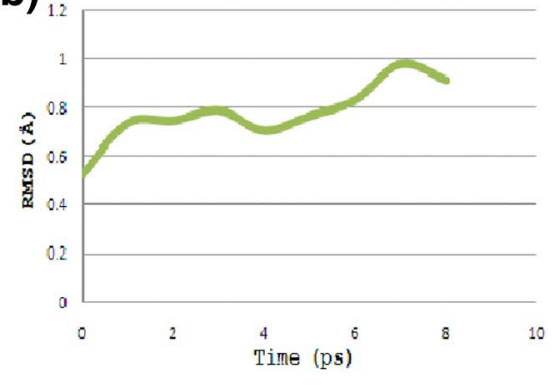

(c)

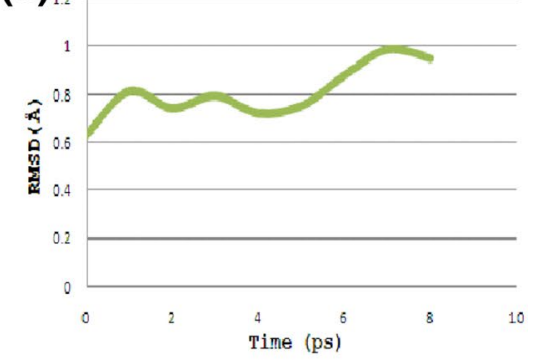

Fig. 7 Graph displaying MD simulation of Nucleocapsid peptide a IRIFVPATN with DRB $1 * 0806$ complex with RMSD highest peak at $0.95 \AA$ A. b IRIFVPATN with DRB1*1304 allele complex with RMSD highest peak at $0.98 \AA$. c MRNLLSQSL and DRB1*0701 allele complex with RMSD highest peak at $0.99 \AA$

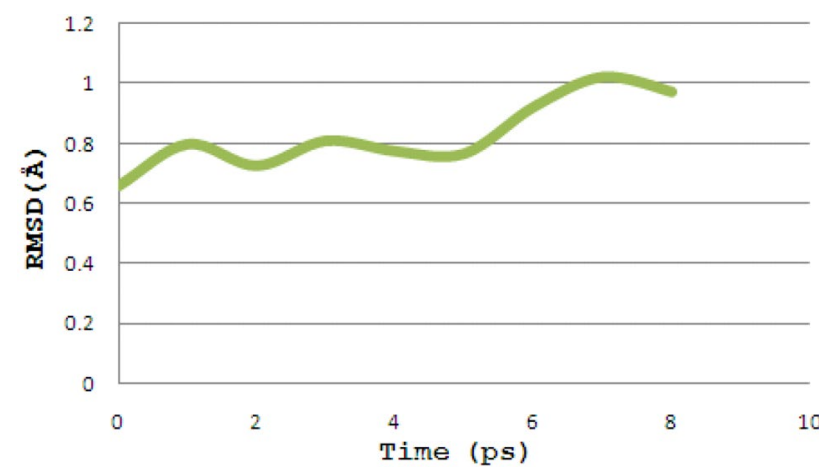

Fig. 8 Graph displaying MD simulation of Matrix peptide VRRAGKYYS and DRB $1 * 0301$ allele complex with RMSD highest peak at $1.02 \AA$

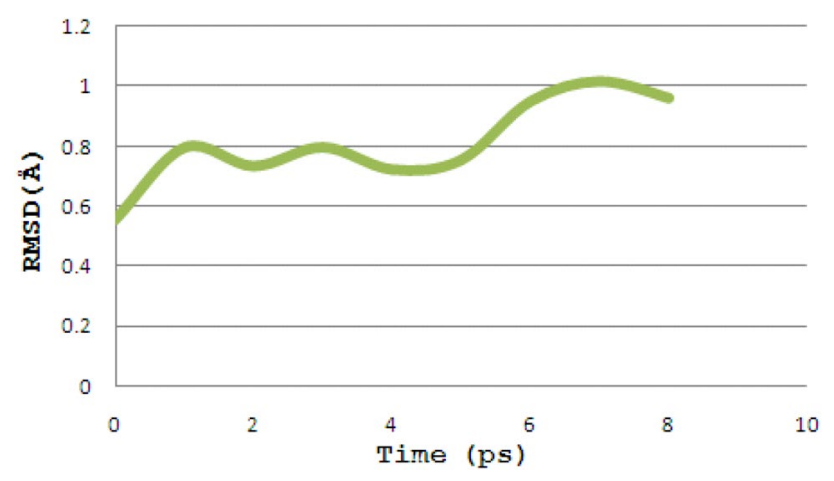

Fig. 9 Graph displaying MD simulation of Fusion peptide VRLKCLLCG with DRB $1 * 0806$ allele complex with RMSD highest peak at $1.01 \AA$

\section{Epitope Conservation and Variability Analysis}

The degree of conservation of epitope in protein sequences provides an important insight about its conservancy through evolution and hence its applicability as an epitope-based vaccine candidate against different strains of the infecting organism. MSA result of best predicted epitopes IRIFVPATN and MRNLLSQSL from nucleocapsid, VRRAGKYYS from Matrix and VRLKCLLCG from Fusion proteins showed that epitopes were well conserved in their entire source protein sequences from different strains of $\mathrm{HeV}$ available at NCBI databank. The MSA results were visualized using Jalview (Fig. 10 a-d). Results were further verified by IEDB epitope conservation analysis tool. It was found that predicted epitopes amino acid sequence were $100 \%$ conserved amongst all the NCBI protein sequence of the source protein of $\mathrm{HeV}$ (Table 3).

\section{Conclusion}

This study identified the potential nanomer T-cell epitopes as a vaccine candidate for Hendra virus. T-cell epitopes IRIFVPATN (nucleocapsid), MRNLLSQSL (nucleocapsid), VRRAGKYYS (matrix) and VRLKCLLCG (fusion) were found to have considerable binding with DRB1*0806, DRB1*1304, DRB1*0701, DRB1*0301 and DRB $1 * 0806$ HLA class II alleles respectively. The chosen epitopes have shown 100\% conservancy throughout all the respective protein sequences from different strains of $\mathrm{HeV}$. These epitopes have shown to have high binding affinity with HLA class II alleles, stable complex formation tendency with HLA class II allele as confirmed by MD simulation results and significant worldwide population coverage. All these results make these epitopes to be a potential candidate for epitope-based vaccine development against $\mathrm{HeV}$ infection. Hence reported epitopes may undergo further in-vivo trials to develop vaccine against $\mathrm{HeV}$ infection. 
Table 8 Estimated Population coverage of predicted $T$ cell epitopes IRIFVPATN, MRNLLSQSL, VRRAGKYYS and VRLKCLLCG based on MHC-I and MHC-II data using IEDB

\begin{tabular}{lllll}
\hline Area/population & \multicolumn{4}{l}{ Epitope (\%) for class I and II coverage } \\
\cline { 2 - 5 } & IRIFVPATN & MRNLLSQSL & VRRAGKYYS & VRLKCLLCG \\
\hline World & $40.24 \%$ & $56.78 \%$ & $36.82 \%$ & $57.08 \%$ \\
East Asia & $32.96 \%$ & $55.25 \%$ & $34.61 \%$ & $49.84 \%$ \\
Northeast Asia & $59.74 \%$ & $66.58 \%$ & $53.24 \%$ & $66.78 \%$ \\
South Asia & $50.55 \%$ & $70.28 \%$ & $49.78 \%$ & $48.22 \%$ \\
Southeast Asia & $50.61 \%$ & $56.60 \%$ & $46.23 \%$ & $60.26 \%$ \\
Southwest Asia & $29.87 \%$ & $44.08 \%$ & $29.28 \%$ & $43.16 \%$ \\
Europe & $41.31 \%$ & $61.57 \%$ & $35.32 \%$ & $59.62 \%$ \\
East Africa & $26.00 \%$ & $25.20 \%$ & $23.80 \%$ & $24.39 \%$ \\
West Africa & $45.81 \%$ & $42.46 \%$ & $28.78 \%$ & $31.79 \%$ \\
Central Africa & $25.19 \%$ & $36.65 \%$ & $26.61 \%$ & $18.14 \%$ \\
North Africa & $29.96 \%$ & $48.82 \%$ & $34.06 \%$ & $36.68 \%$ \\
South Africa & $39.42 \%$ & $33.74 \%$ & $42.58 \%$ & $21.14 \%$ \\
West Indies & $35.01 \%$ & $49.11 \%$ & $19.93 \%$ & $42.58 \%$ \\
North America & $35.23 \%$ & $56.04 \%$ & $37.08 \%$ & $57.29 \%$ \\
Central America & $0.77 \%$ & $11.22 \%$ & $3.65 \%$ & $0.00 \%$ \\
South America & $29.76 \%$ & $46.12 \%$ & $27.22 \%$ & $37.50 \%$ \\
Oceania & $40.20 \%$ & $44.67 \%$ & $37.35 \%$ & $47.43 \%$ \\
Average (standard deviation) & $36.04 \%(12.70 \%)$ & $47.36 \%(14.46 \%)$ & $33.31 \%(11.48 \%)$ & $41.29 \%(17.27 \%)$ \\
\hline
\end{tabular}

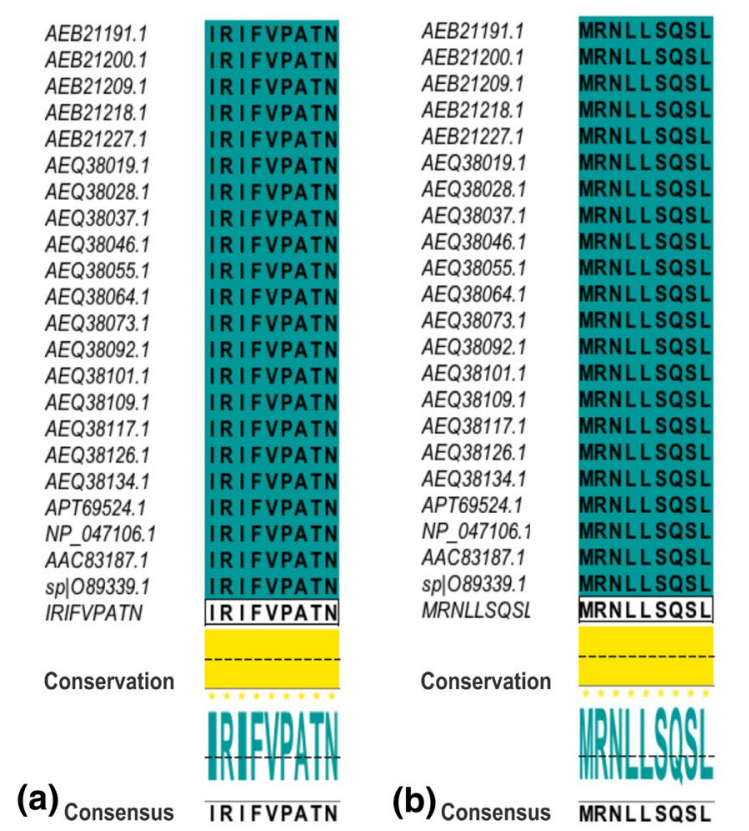

Fig. 10 Degree of Conservancy using MSA showed that Epitopes a IRIFVPATN (Nucleocapsid) b MRNLLSQSL (Nucleocapsid) c VRRAGKYYS (Matrix) d VRLKCLLCG (Fusion) were 100\% con-

Acknowledgements The authors are grateful for the necessary immunoinformatic facilities and support provided by the faculty members of Dept. of Zoology, Government P. G. College, Guna \& Dept of Microbiology, College of Life Science, Cancer Hospital Campus Gwalior, Madhya Pradesh, India.

\begin{tabular}{|c|c|c|c|}
\hline AEB21208.1 & VRRAGKYYS & AEB21197.1 & VRLKCLLCG \\
\hline AEB21214.1 & VRRAGKYYS & AEB21205.1 & VRLKCLLCG \\
\hline AEB21223.1 & VRRAGKYYS & AEB21215.1 & VRLKCLLCG \\
\hline AEB21232.1 & VRRAGKYYS & AEB21224.1 & VRLKCLLCG \\
\hline AEQ38024.1 & VRRAGKYYS & AEB21233.1 & VRLKCLLCG \\
\hline AEQ38033.1 & $\begin{array}{l}\text { VRRAGKYS } \\
\text { VRR }\end{array}$ & AEQ38025.1 & VRLKCLLCG \\
\hline$A E Q 38041.1$ & VRRAGKYYS & AEQ38034.1 & VRLKCLLCG \\
\hline AEQ38050.1 & VRRAGKYYS & AEQ38042.1 & VRLKCLLCG \\
\hline AEQ38060.1 & VRRAGKYYS & AEQ38051.1 & VRLKCLLCG \\
\hline AEQ38069.1 & VRRAGKYYS & AEQ38061.1 & VRLKCLLCG \\
\hline$A E Q 38078.1$ & VRRAGKYYS & AEQ38070.1 & VRLKCLLCG \\
\hline AAC83191.2 & VRRAGKYYS & AEQ38079.1 & VRLKCLLCG \\
\hline NP 047110.2 & & AEQ38098.1 & VRLKCLLCG \\
\hline$A E Q 38097.1$ & $\begin{array}{l}\text { VRKAGH } \\
\text { VRRAGKYYS }\end{array}$ & AEQ38107.1 & VRLKCLLCG \\
\hline AEQ38106.1 & VRRAGKYYS & $\begin{array}{l}A E Q 38114.1 \\
A E Q 38123.1\end{array}$ & $\begin{array}{l}\text { VRLKCLLCG } \\
\text { VRLKLLC }\end{array}$ \\
\hline AEQ38113.1 & VRRAGKYYS & AEQ38132.1 & VRLKCLLCG \\
\hline AEQ38122.1 & VRRAGKYYS & AEQ38140.1 & VRLKCLLCG \\
\hline AEQ38131.1 & VRRAGKYYS & APT69529.1 & VRLKCLLCG \\
\hline AEQ38139.1 & VRRAGKYYS & $A A B 39505.1$ & VRLKCLLCG \\
\hline APT69528.1 & VRRAGKYYS & NP_047111.2 & VRLKCLLCG \\
\hline VRRAGKYYS & VRRAGKYYS & $A A \bar{C} 83192.2$ & VRLKCLLCG \\
\hline & & VRLKCLLCG & VRL \\
\hline \multirow[t]{3}{*}{ Conservation } & & \multirow{3}{*}{ Conservation } & \\
\hline & & & \\
\hline & & & \\
\hline & VRRAGKYYS & (d) Consensus & VRLKCLLCG \\
\hline
\end{tabular}

served in all the protein sequences obtain from different isolated $\mathrm{HeV}$ strains worldwide. Yellow colored rectangle shows conserved nanomer epitopes

Funding This research did not receive any specific grant from funding agencies in the public, commercial, or not-for-profit sectors. 


\section{Compliance with Ethical Standards}

Conflict of interest Authors declares that they have no conflict of interest.

Ethical Approval This article does not contain any studies with human participants or animals performed by any of the authors.

\section{References}

Altschul SF, Gish W, Miller W, Myers EW, Lipman DJ (1990) Basic local alignment search tool. J Mol Biol 215(3):403-410

Bui HH, Sidney J, Dinh K, Southwood S, Newman MJ, Sette A (2006) Predicting population coverage of T-cell epitope-based diagnostics and vaccines. BMC Bioinform 7(1):153

Bui HH, Sidney J, Li W, Fusseder N, Sette A (2007) Development of an epitope conservancy analysis tool to facilitate the design of epitope-based diagnostics and vaccines. BMC Bioinform 8(1):361

Doytchinova IA, Flower DR (2007) VaxiJen: a server for prediction of protective antigens, tumour antigens and subunit vaccines. BMC Bioinform 8(1):4

Gonzalez-Galarza FF, Christmas S, Middleton D, Jones AR (2010) Allele frequency net: a database and online repository for immune gene frequencies in worldwide populations. Nucleic Acids Res 39(suppl_1):D913-D919

Guan P, Doytchinova IA, Zygouri C, Flower DR (2003) MHCPred: a server for quantitative prediction of peptide-MHC binding. Nucleic Acids Res 31(13):3621-3624

Gupta S, Kapoor P, Chaudhary K, Gautam A, Kumar R, Raghava GP, Open Source Drug Discovery Consortium (2013) In silico approach for predicting toxicity of peptides and proteins. PLoS ONE 8(9):e73957

Halpin K, Young PL, Field HE, Mackenzie JS (2000) Isolation of Hendra virus from pteropid bats: a natural reservoir of Hendra virus. J Gen Virol 81(8):1927-1932

Hanna JN, McBride WJ, Brookes DL, Shield J, Taylor CT, Smith IL, Craig SB, Smith GA (2006) Hendra virus infection in a veterinarian. Med J Aust 185:562-564

Humphrey W, Dalke A, Schulten K (1996) VMD: visual molecular dynamics. J Mol Graph 14(1):33-38

Jones DT (1999) Protein secondary structure prediction based on position-specific scoring matrices. J Mol Biol 292(2):195-202

Kamthania M, Sharma DK (2016) Epitope-Based Peptides Prediction from Proteome of Nipah Virus. Int J Pept Res Ther 22(4):465-470

Kaur H, Raghava GPS (2004) A neural network method for prediction of $\beta$-turn types in proteins using evolutionary information. Bioinformatics 20(16):2751-2758

Laskowski RA, MacArthur MW, Moss DS, Thornton JM (1993) PROCHECK: a program to check the stereochemical quality of protein structures. J Appl Crystallogr 26(2):283-291

Li W, Joshi MD, Singhania S, Ramsey KH, Murthy AK (2014) Peptide vaccine: progress and challenges. Vaccines 2(3):515-536
Mire CE, Satterfieled BA, Geisbert TW (2015) Pathogenesis of Hendra Virus in Humans. In: Singh SK (ed) Human Emerging and Re-emerging Infections: viral and parasitic infections, Volume II pp 207-226

Morris GM, Goodsell DS, Halliday RS, Huey R, Hart WE, Belew RK, Olson AJ (1998) Automated docking using a Lamarckian genetic algorithm and an empirical binding free energy function. J Comput Chem 19(14):1639-1662

Murray K, Rogers R, Selvey L, Selleck P, Hyatt A, Gould A, Gleeson L, Hooper P, Westbury H (1995) A novel morbillivirus pneumonia of horses and its transmission to humans. Emerg Infect Dis $1(1): 31$

O'sullivan JD, Allworth AM, Paterson DL, Snow TM, Boots R, Gleeson LJ, Gould AR, Hyatt AD, Bradfield J (1997) Fatal encephalitis due to novel paramyxovirus transmitted from horses. The Lancet 349(9045):93-95

Phillips JC, Braun R, Wang W, Gumbart J, Tajkhorshid E, Villa E, Chipot C, Skel RD, Kale L, Schulten K (2005) Scalable molecular dynamics with NAMD. J Comput Chem 26(16):1781-1802

Playford EG, McCall B, Smith G, Slinko V, Allen G, Smith I, Field H (2010) Human Hendra virus encephalitis associated with equine outbreak. Australia 2008 Emerg Infect Dis 16(2):219

Robinson J, Halliwell JA, McWilliam H, Lopez R, Parham P, Marsh SG (2012) The imgt/hla database. Nucleic Acids Res 41(D1):D1222-D1227

Šali A, Potterton L, Yuan F, van Vlijmen H, Karplus M (1995) Evaluation of comparative protein modeling by MODELLER. Proteins: Structure, Function, and Bioinformatics 23(3):318-3265

Sanner MF (1999) Python: a programming language for software integration and development. J Mol Graph Model 17(1):57-61

Selvey LA, Wells RM, McCormack JG, Ansford AJ, Murray K, Rogers RJ, Lavercombe PS, Selleck P, Sheridan JW (1995) Infection of humans and horses by a newly described morbillivirus. Med J Aust 162(12):642-644

Sievers F, Wilm A, Dineen D, Gibson TJ, Karplus k, Li W, Lopez R, McWilliam H, Remmert M, Söding J, Thompson JD, Higgins DG (2011) Fast, scalable generation of high-quality protein multiple sequence alignments using clustal omega. Mol Syst Biol 7:539

Singh H, Raghava GPS (2001) ProPred: prediction of HLA-DR binding sites. Bioinformatics 17(12):1236-1237

Singh S, Singh H, Tuknait A, Chaudhary K, Singh B, Kumaran S, Raghava GP (2015) PEPstrMOD: structure prediction of peptides containing natural, non-natural and modified residues. Biol Direct 10(1):73

Waterhouse AM, Procter JB, Martin DM, Clamp M, Barton GJ (2009) Jalview Version 2-a multiple sequence alignment editor and analysis workbench. Bioinformatics 25(9):1189-1191

Publisher's Note Springer Nature remains neutral with regard to jurisdictional claims in published maps and institutional affiliations. 